\title{
О ПОСТРОЕНИИ БЫСТРО СХОДЯЩИХСЯ МЕТОДОВ НЕГЛАДКОЙ ОПТИМИЗАЦИИ МНОГОСЛОЙНОГО ПЕРСЕПТРОНА
}

\author{
А. И. Каплинский, А. М. Песин \\ Воронежский государственный университет
}

Поступила в редакцию 01.12.2018 г.

\begin{abstract}
Аннотация. В статье предлагается подход к построению поисковых методов, обладающих высокой скоростью сходимости, для оптимизации критерия качества настройки многослойного персептрона. Рассматриваемая задача имеет непосредственное отношение к построению искусственных нейронных сетей и разработке нейрокомпьютеров.

Ключевые слова: персептрон, условие локального улучшения, потенциал.

Annotation. The paper proposes an approach to the construction of search methods with a high convergence rate to optimize the quality criterion of setting a multilayer perceptron. The considered problem is directly related to the building of artificial neural networks and the development of neural computers.
\end{abstract}

Keywords: perceptron, condition of local improvement, potential.

\section{ВВЕДЕНИЕ}

В работах [1-5] предложены и исследованы методы оптимизации скалярных негладких и многоэкстремальных функций векторного аргумента. В [7] данный подход развивается для построения быстро сходящихся методов негладкой и нелокальной оптимизации. В $[6,8]$ изучались вопросы выхода оптимизируемой функции из области локального экстремума. В настоящей работе предложенный подход используется для решения задачи идентификации на примере оптимизации многослойного персептрона [12]. Задача оптимизации многослойного персептрона имеет непосредственное отношение к построению вычислительных схем, названных искусственными нейросетями $[9,10,12]$, и построению вычислительных устройств, названных нейрокомпьютерами $[9,10,12]$.

(с) Каплинский А. И., Песин А. М., 2018

\section{1. ПОСТАНОВКА И АНАЛИЗ ЗАДАЧИ}

Пусть требуется минимизировать скалярную, возможно негладкую и многоэкстремальную функцию $\Phi[Y]$ векторного аргумента $Y, n \geq 1, Y \in R^{n}$. Функция $\Phi[Y]$ является критерием качества настройки многослойного персептрона [12].

Векторы входов $Y_{N}$ имеют следующий вид:

$$
Y_{N}=f\left[W_{N} Y_{N-1}\right],
$$

где $N \geq 1, f=\left(f_{1}, \ldots, f_{n}\right), f_{i}(i=\overline{1, n})$ - нелинейные сигмоидные отображения [10, 12]. Здесь $W_{N}(N \geq 1)$ - это числовые матрицы размерности $n \times n$, которые выбираются с целью решения следующей задачи оптимизации

$$
\Phi\left[f\left[W_{N} Y_{N-1}\right]\right] \rightarrow \min _{\left(W_{N}, W_{N-1}, \ldots, W_{0}\right)} .
$$

Введем обозначение $z_{N}=W_{N} Y_{N-1}$, где $N \geq 1$. Тогда настраиваемая модель со структурой многослойного персептрона имеет вид

$$
\Phi\left[f\left(z_{N}\right)\right] \rightarrow \Phi\left[Y_{N}\right]_{\left(W_{N}, W_{N-1}, \ldots, W_{0}\right)} .
$$




\section{А. И. Каплинский, А. М. Песин}

В частном случае можно рассматривать минимизацию среднеквадратического критерия качества вида

$$
\Phi\left[Y_{N}\right] \rightarrow M\left[Y_{N}-Y^{*}\right]^{2} \min _{\left(W_{N}, W_{N-1}, \ldots, W_{0}\right)},
$$

где матрицы $W_{N}$ настраиваемой модели (2) могут выбираться стохастическими [11].

Задача (1)-(2) может рассматриваться как задача идентификации заданного вектора входов $Y^{*}$ векторами выходов $Y_{N}$ настраиваемой модели (2), при этом вектор входов $Y^{*}$ также может считаться случайным. В данной статье рассматривается задача (1)-(2) применительно к возможно негладкой и многоэкстремальной функции $\Phi[Y]: R^{n} \rightarrow R^{1}$, причем упор делается на построение именно поисковых методов оптимизации. Этот выбор обусловлен тем, что согласно $[9,10,12]$, вектор-функция $f=\left(f_{1}, \ldots, f_{n}\right)$ состоит из сигмоидных нелинейных одномерных отображений $f_{i}[9,10,12]$. При использовании методов гладкой оптимизации в случае, например, критерия $\Phi\left[Y_{N}\right] \rightarrow M\left[Y_{N}-Y^{*}\right]^{2}$ с необходимостью участвует производная $f_{i}^{\prime}$ сигмоидного отображения. Дифференцирование отображений $f_{i}$ ввиду их структуры приводит к обнулению достаточно больших массивов коэффициентов матриц $W_{N}$, что может привести к отсутствию работоспособности настраиваемого многослойного персептрона $[9,10,12]$.

\section{2. ПОСТРОЕНИЕ МЕТОДОВ РЕШЕНИЯ ЗАДАЧИ (1)-(2)}

Согласно работам $[1,4,5,11]$, построение эффективных на практике методов негладкой оптимизации многоэкстремальных функций заключается в предварительной рандомизации исходной задачи (1)-(2). Для этого рассмотрим матричный линейный стохастический процесс

$$
\left\{\begin{array}{l}
W_{N}=W_{N-1}+\varepsilon_{N} \theta_{N}(N \geq 1) \\
\left\|M\left(W_{0}\right)\right\|<\infty,\left\|\left(\theta_{1}\right)\right\|<\infty, 0<\varepsilon_{N}<\infty \\
\left(\varepsilon_{1}+\varepsilon_{2}+\ldots\right)=\infty
\end{array}\right.
$$

Согласно введенным ранее обозначениям, имеет место выражение:

$$
Z_{N}=W_{N} Y_{N-1}+\varepsilon_{N} \cdot \theta_{N} \cdot Y_{N-1} .
$$

Векторный поисковый случайный процесс (4) приводит к рандомизации следующего критерия оптимизации:

$$
F\left(\varepsilon_{N}\right)=\int_{R^{N}} \hat{\Phi}[f(z)] p_{Z_{N}}(z) d z,
$$

где $\hat{\Phi}[f(z)]=\Phi[f(z)]-c_{N}, c_{N}=$ const.

Задача (1)-(2) преобразуется в следующую задачу:

$$
F\left(\varepsilon_{N}\right) \rightarrow \min _{\left(\varepsilon_{N}\right)} .
$$

Заметим, что разложение (4) $Z_{N}$ в сумму двух случайных векторов позволяет полностью обосновать математическую корректность перехода от задачи (1)-(2) к задаче (5) [например, $1,3,4]$.

Предположим, что существует плотность распределения $p_{Z_{V}}$ случайного вектора $Z_{N}$ для каждого $N=1,2,3, \ldots$. Пусть выполняется условие знакоопределенности

$$
\hat{\Phi}[f(z)]=\Phi[f(z)]-c_{N} \geq 0,
$$

где $c_{N}$ - соответствующие константы, такие что $0<c_{N}<\infty,(N=1,2,3, \ldots)$.

При заданном на шаге $N$ векторе входов $Y_{N-1}$ должна выбираться из условия (6) матрица $\theta_{N}$, задающая вектор $\theta_{N} Y_{N-1}$ направления движения для решения задач (1)-(2) или (5). Условие невозрастания критерия оптимизации $F\left(\varepsilon_{N}\right)$ на каждом шаге $N=1,2,3, \ldots$ названо условием локального улучшения (УЛУ) $[1,2,3,4]$. Оно имеет следующий вид:

$$
\left.\frac{d F}{d \varepsilon}\right|_{\varepsilon=0} \leq 0 .
$$

Для задач (1)-(2) и (5) УлУ (6) имеет вид

$$
\begin{gathered}
\left.\frac{d F}{d \varepsilon}\right|_{\varepsilon=0} \leq 0=\int_{R^{N}} \hat{\Phi}[f(z)] \cdot d i v_{Z}\left[p_{W_{N-1} Y_{N-1}}(z) \times\right. \\
\times M\left(\theta_{N} Y_{N-1} / W_{N-1} Y_{N-1}=z\right) d z,
\end{gathered}
$$

где $M\left(\theta_{N} Y_{N-1} / W_{N-1} Y_{N-1}=z\right)$ - вектор условного математического ожидания случайного вектора $\theta_{N} Y_{N-1}$.

Для дальнейших рассуждений введем обозначение $y_{N}(z)=M\left(\theta_{N} Y_{N-1} / W_{N-1} Y_{N-1}=z\right)$.

С целью выполнения УЛУ (7) выберем вектор-функцию $y_{N}(z)$ как решение следующего уравнения:

$$
\begin{aligned}
& d i v_{Z}\left[p_{W_{N-1} Y_{N-1}}(z) \cdot y_{N}(z)\right]= \\
& =-p_{u_{N}}(z)\left[\Phi[f(z)]-c_{N}\right],
\end{aligned}
$$


при этом параметры $c_{N}$, названные константами уровней, выбираются из условия «неразрывности» $[4,5,11,8]$

$$
\int_{R^{N}} \operatorname{div}_{Z}\left[p_{W_{N-1} Y_{N-1}}(z) \cdot y_{N}(z)\right] d z=0 .
$$

В (8) функция $p_{u_{N}}(z)$ имеет смысл плотности распределения вероятности вспомогательного случайного вектора $u_{N}(N=1,2,3, \ldots)$, функция $p_{u_{N}}(z)$ определяет степень свободы при решении уравнения (8) $[3,5,1,8,11]$.

При некоторых условиях $[1,2,3,4]$ векторное поле $p_{W_{N-1} Y_{N-1}}(z) \cdot y_{N}(z)$ можно разложить следующим образом

$$
p_{W_{N-1} Y_{N-1}}(z) \cdot y_{N}(z)=\nabla_{Z} v_{N}(z)+\chi_{N}(z) .
$$

Здесь $v_{N}(z): R^{n} \rightarrow R^{1}$ для каждого значения $N=1,2,3, \ldots$ является потенциалом векторного поля $p_{W_{N-1} Y_{N-1}}(z)$, а $\chi_{N}(z): R^{n} \rightarrow R^{1}$ есть бездивергентная составляющая разложения, то есть $d i v_{Z}\left[\chi_{N}(z)\right]=0$. Составляющая $\chi_{N}(z)$ определяет степень свободы в (9), причем считается, что $\chi_{N}(z)=0,(N=1,2,3, \ldots)$.

Подставляя разложение (9) в уравнение (8), получим уравнение Пуассона на потенциале $v_{N}(z)$ вила

$$
\Delta_{Z} v_{N}(z)=-p_{u_{N}}(z)[\hat{\Phi}[f(z)]],
$$

где $\Delta=\sum_{i=1}^{n} \frac{\partial^{2}}{\partial x_{i}^{2}}$.

Решение уравнения (10) имеет следующий вид

$$
v_{N}(z)=\int_{R^{N}} \mathrm{E}(z, s) \hat{\Phi}[f(s)] \cdot p_{u_{N}}(s) d s,
$$

где $E(z, s)=\left\{\begin{array}{l}(n-2)^{-1} \cdot \omega_{n}^{-1}|z-s|^{2-n}, n>2, \\ \frac{1}{2 \pi} \log |z-s|^{-1}, n=2 ;\end{array}\right.$

$\omega_{n}=\frac{2(\sqrt{\pi})^{n}}{\Gamma(n / 2)}-$ площадь поверхности единичной сферы в $R^{n}$.

Таким образом, функция (11) является потенциалом ньютоновского векторного поля (9) [1-4]. С целью ускорения сходимости предлагаемых методов оптимизации в работе [7] развивался подход, связанный с использованием собственных функций линейных операторов. Применим здесь этот подход и получим другой способ выполнения УЛУ (7).
В условиях [1-4] векторное поле $p_{u_{N}}(z) y_{N}(z)$ можно представить следующим образом

$$
p_{u_{N}}(z) y_{N}(z)=\nabla_{Z}\left[\varphi_{N}(z)\right],
$$

где

$$
\begin{gathered}
\Delta \varphi_{N}+q_{N}^{2} \varphi_{N}= \\
=-p_{u_{N}}(z)[\hat{\Phi}[f(z)]], N=1,2,3, . .
\end{gathered}
$$

$0<q_{N}<\infty-$ константы.

Потенциал $\varphi_{N}(z)$ является решением уравнения (13) типа Гельмгольца и имеет вид

$$
\varphi_{N}(z)=\int_{R^{N}} \mathrm{E}_{q}(z, s) \hat{\Phi}[f(s)] \cdot p_{u_{N}}(s) d s .
$$

В решении (14) ядро $\mathrm{E}_{q}(z, s)$ - это сферически-инвариантное (или фундаментальное) решение уравнения $\left(\nabla_{Z}+q_{N}^{2}\right) \mathrm{E}_{q}=0[7,8]$.

Функция $\mathrm{E}_{q}(z, s)$ имеет следующий вид:

a) для четных $n$

$$
\mathrm{E}_{q}(z, s)=|z-s|^{-\frac{(n-2)}{2}} \cdot J_{-\frac{(n-2)}{2}}\left(q_{N}|z-s|\right),
$$

б) для нечетных $n$

$$
\mathrm{E}_{q}(z, s)=|z-s|^{-\frac{(n-2)}{2}} \cdot J_{\frac{(n-2)}{2}}\left(q_{N}|z-s|\right) .
$$

В выражении (15) $J_{-\beta}$ и $N_{\beta}$ обозначают соответствующие функции Бесселя и Неймана $\beta$-го порядка $[7,8]$.

Таким образом, функция $\varphi_{N}(z)(14)$ является потенциалом векторного поля (12).

\section{3. УСКОРЕНИЕ СХОДИМОСТИ}

Выберем функцию $p_{u_{N}}(z)$ в (13) как решение интегрального уравнения

$$
\begin{gathered}
\hat{\Phi}[f(z)] p_{u_{N}}(z)= \\
=U_{N}(z) \int_{R^{N}} \mathrm{E}_{q}(z, s) \hat{\Phi}[f(s)] \cdot p_{u_{N}}(s) d s,
\end{gathered}
$$

где $U_{N}(z): R^{n} \rightarrow R^{1}$.

Тогда, учитывая (14), получим следующее равенство

$$
\hat{\Phi}[f(z)] p_{u_{N}}(z) d z=-U_{N}(z) \varphi_{N}(z) .
$$

Тогда уравнение (13) перепишется в виде

$$
\Delta \varphi_{N}+q_{N}^{2} \varphi_{N}=U_{N} \varphi_{N}, N=1,2,3 \ldots
$$

Уравнение (17) при определенном способе выбора констант $q_{N}$ и функций $U_{N}$ представляет собой уравнение Шредингера на 


\section{А. И. Каплинский, А. М. Песин}

стационарные состояния системы с «потенциальной энергией» $U_{N}(z)$.

Выберем теперь функцию $U_{N}(z)$ на каждом шаге $N=1,2,3, \ldots$ таким образом, чтобы обеспечить ускорение сходимости конструируемых методов оптимизации. Для этого потребуем выполнения «соотношения оптимальности» для функций $\varphi_{N}(z)$

$$
\hat{\Phi}[f(z)]=\varphi_{N}(z) .
$$

Тогда из формул (14)-(16) получим $U_{N}(z)=p_{u_{N}}(z)_{N}$. Соотношение оптимальности (18) означает, что точки, где расположены экстремумы функции $\hat{\Phi}[f(z)]$, совпадают с точками расположения экстремумов функции $\varphi_{N}(z)$ для всех $N$. Соотношение (18) может выполняться лишь в окрестностях точек, где функция $\hat{\Phi}[f(z)]$ обладает для этого достаточной гладкостью. В других точках пространства $R^{n}$ соотношение (18) является оптимальным сглаживанием функции $\hat{\Phi}[f(z)]$ [7]. Таким образом, для ускорения сходимости конструируемых методов оптимизации предлагается выбирать степени свободы $p_{u_{N}}(z)$ в (14) как решение интегрального уравнения (16) с учетом «соотношения оптимальности» (18).

В результате получим интегральное уравнение Фредгольма для выбора оптимальной функции $p_{u}^{*}(s)$

$$
\hat{\Phi}[f(z)]=\int_{R^{N}} \mathrm{E}_{q}(z, s) \hat{\Phi}[f(s)] p_{u}^{*}(s) d s .
$$

Отметим, что получение решения $p_{u}^{*}(s)$ уравнения (19) требует привлечения входной информации лишь о значениях оптимизируемой функции $\hat{\Phi}[f(z)]$. Затем функция $p_{u}^{*}(s)$ подставляется в (14) и получается оптимальный потенциал $\varphi^{*}(z)$ вида

$$
\varphi^{*}(z)=-\int_{R^{N}} \mathrm{E}_{q}(z, s) \hat{\Phi}[f(s)] p_{u}^{*}(s) d s .
$$

\section{4. МЕТОДЫ ОПТИМИЗАЦИИ ДЛЯ РЕШЕНИЯ ЗАДАЧИ 1-2}

Многослойный персептрон (2) настраивается процессом (3), где матрицы $\theta_{N}$, задающие «направления матричного движения», определяются своими условными математи- ческими ожиданиями $y_{N}(z)$ (9) или (12) на каждом шаге $N=1,2,3 \ldots$

Из соотношения (9) следует, что

$$
y_{N}(z)=p_{W_{N-1} Y_{N-1}}^{-1}(z) \nabla_{z} v_{N}(z),
$$

где $v_{N}(z)$ задается формулой (11). Из соотношения (12) следует другое решение

$$
y^{*}(z)=\left(p_{u}^{*}(z)\right)^{-1} \nabla_{z} \varphi^{*}(z)
$$

где функция $p_{u}^{*}(z)$ является решением уравнения (19), а функция $\varphi^{*}(z)$ задается формулой (20). Решение $y^{*}(z)$ используется с целью получения методов оптимизации, обладающих быстрой сходимостью $[7,8]$.

Настройка констант уровня $c_{N}$ проводится в соответствии с условием «неразрывности» и имеет следующий вид $[4,5,11]$

$$
\left\{\begin{aligned}
& c_{N}= \int_{R^{N}} \hat{\Phi}[f(s)] p_{u_{N}}(s) d s=M\left[\hat{\Phi}\left(f\left(u_{N}\right)\right)\right], \\
& c^{*}=\int_{R^{N}} \hat{\Phi}[f(s)] p_{u}^{*}(z) d s=M[\hat{\Phi}(f(u))], \\
& \quad N=1,2,3 \ldots
\end{aligned}\right.
$$

Уравнения «типа регрессии» (21) могут быть решены, например, следующим образом

$$
\left\{\begin{array}{l}
c_{N, K}=c_{N, K}+\frac{1}{k}\left\{\hat{\Phi}\left[f\left(s_{N, K}\right)\right]\right\}-c_{N, K-1}, \\
c_{K}^{*}=c_{K-1}^{*}+\frac{1}{k}\left\{\hat{\Phi}\left[f\left(s_{K}^{*}\right)\right]-c_{K-1}^{*}\right\},
\end{array}\right.
$$

где $(k=1,2, \ldots), \quad\left|c_{1,0}\right|<\infty, \quad\left|c_{0}\right|<\infty$.

В формулах (22) через $c_{N, K}$ и $c_{K}^{*}$ обозначены оценки констант уровня $c_{N}$ и $c^{*}$ соответственно, через $s_{N, K}$ и $s_{K}^{*}$ обозначены реализации случайных векторов $u_{N}$ и $u^{*}$ с плотностями распределений $p_{u_{N}}(s)$ и $p_{u}^{*}(s), k=1,2, \ldots$ для каждого $N$.

Аппроксимируем условное математическое ожидание одной реализацией соответствующего случайного вектора

$$
\begin{aligned}
& y_{N}(z)=M\left[\theta_{N} Y_{N-1} / W_{N-1} Y_{N-1}=z\right] \approx \theta_{N} Y_{N-1} . \\
& \text { Тогда } \\
& \qquad \theta_{N} Y_{N-1} \approx \nabla_{z} v_{N}(z) p_{W_{N-1} Y_{N-1}}^{-1}(z),
\end{aligned}
$$

где $z=W_{N-1} Y_{N-1}$.

Соответственно получим

$$
\theta_{N}^{*} Y_{N-1} \approx \nabla_{z} \varphi^{*}(z)\left[p_{W_{N-1}^{*} Y_{N-1}}^{-1}(z)\right]^{-1},
$$

где $z=W_{N-1}^{*} Y_{N-1}$.

Из соотношений (23), (24) получаются ма- 
трицы $\theta_{N}$ и $\theta_{N}^{*}$ вида

$$
\left\{\begin{array}{l}
\theta_{N} \approx\left[Y_{N}^{T} Y_{N}\right]^{-1}\left[Y_{N}^{T} \nabla_{z} v_{N}(z)\right] p_{W_{N-1} Y_{N-1}}^{-1}(z), \\
\theta_{N}^{*} \approx\left[Y_{N}^{T} Y_{N}\right]^{-1}\left[Y_{N}^{T} \nabla_{z} \varphi^{*}(z)\right]\left(p_{W_{N-1}^{*} Y_{N-1}}^{-1}(z)\right)^{-1} .
\end{array}\right.
$$

Таким образом, получены следующие формулы, позволяющие организовать оптимизационную процедуру

$$
\text { a) } \begin{aligned}
& W_{N}=W_{N-1}+\varepsilon_{N}\left[Y_{N}^{T} Y_{N}\right]^{-1} \times \\
& \times\left[Y_{N}^{T} \nabla_{z} v_{N}(z)\right] p_{W_{N-1} Y_{N-1}}^{-1}(z),
\end{aligned}
$$

где $z=W_{N-1} Y_{N-1}, \quad Y_{i}=f\left(W_{i-1} Y_{i-1}\right), \quad(i=1,2,3, \ldots)$; $\left\|M\left(W_{1}\right)\right\|<\infty$; потенциал $v_{N}(z)$ задается формулой (11),

$\nabla_{z} v_{N}(z)=\int_{R^{N}} p_{u_{N}}(s) \nabla_{z} E(z, s)\left[\Phi(f(s))-c_{N}\right] d s ;$

$c_{N}$ определяется процедурой (22);

$$
\begin{aligned}
& \text { б) } W_{N}^{*}=W_{N-1}^{*}+\varepsilon_{N}\left[Y_{N}^{T} Y_{N}\right]^{-1} \times \\
& \times\left[Y_{N}^{T} \nabla_{z} \varphi^{*}\left(z^{*}\right)\right] p_{W_{N-1}^{*} Y_{N-1}^{*}}^{-1}\left(z^{*}\right),
\end{aligned}
$$

где $z^{*}=W_{N-1}^{*} Y_{N-1}, \quad Y_{i}=f\left(W_{i}^{*} Y_{i-1}\right) \quad(i=1,2,3, \ldots)$; $\left\|M\left(W_{1}^{*}\right)\right\|<\infty$; оптимальный потенциал $\varphi^{*}(z)$ задан формулой (20);

$\nabla_{z} \varphi^{*}(z)=\int_{R^{N}} \nabla_{z} E_{q}(z, s) p_{u}^{*}(s)\left[\hat{\Phi}(f(s))-c^{*}\right] d s ;$

$c^{*}$ определяется процедурой (22).

Выбор числовой последовательности $\varepsilon_{N}$ в рамках условий процедуры (3) может производиться различными способами $[4,5,3,11]$.

В качестве примера в [11] был предложен следующий способ

$$
\varepsilon_{N+1}=\left\{\begin{array}{l}
\varepsilon_{N} \gamma_{1}, \text { если } \hat{\Phi}\left(f\left(z_{N}\right)\right)<\hat{\Phi}\left(f\left(z_{N-1}\right)\right), \\
\varepsilon_{N} \gamma_{2}, \text { если } \hat{\Phi}\left(f\left(z_{N}\right)\right) \geq \hat{\Phi}\left(f\left(z_{N-1}\right)\right),
\end{array}\right.
$$

где $0<\gamma_{1}<1 ; 0<\gamma_{2}<1 ; \gamma_{1}$ выбирается эмпирически; $\gamma_{2}$ выбирается с учетом $\gamma_{1}$ из соотношения $\ln \gamma_{2}=\lambda \ln \gamma_{1}$, где $\lambda<0$.

Последовательность $\varepsilon_{N}$ должна с необходимостью удовлетворять (3).

Условия сходимости и другие свойства методов оптимизации типа (26), (27) рассматривались в работах $[7,11,8,6]$.

\section{ЗАКЛЮЧЕНИЕ}

Основными преимуществами предлагаемых методов оптимизации (26), (27) перед традиционными, согласно [7, 11, 5, 8, 6, 4], являются их возможности, связанные с заменой функции $\hat{\Phi}$ на потенциалы $v$ и $\varphi$. Это позволило сконструировать градиентные методы (26) и (27) в негладкой задаче оптимизации (1)-(2). Здесь оптимальная настройка многослойного персептрона в смысле решения задачи (2) осуществляется процессом перестройки матриц $W_{N}$ и $W_{N}^{*}$. Остальные соотношения являются необходимыми для построения таких матричных процессов. Структура многослойного персептрона (2) входит в (26) и (27) через аргументы $z$ и $z^{*}$ и плотности распределения $p_{W_{N-1} Y_{N-1}}(z)$ и $p_{W_{N-1}^{Y} Y_{N-1}}^{*}(z)$. Поскольку при построении (27) использовалось соотношение оптимальности (18), то возможно достижение высокой скорости сходимости оценок (27) к своим предельным значениям.

В методах (26) и (27) не используется градиент функции $\hat{\Phi}$, поэтому по отношению к ней они являются поисковыми методами оптимизации. Для подхода, развитого в работах $[3,4,5,6,7,8,11]$, характерно, что предлагаемые методы негладкой и нелокальной оптимизации могут использовать лишь информацию о значениях оптимизируемой функции $\hat{\Phi}$. Таким же свойством обладают и методы (26) и (27). Эти обстоятельства лишают предлагаемые методы недостатков, связанных с дифференцированием сигмоидных отображений и обнулением больших массивов матриц $W_{N},(n=1,2,3, \ldots)$.

\section{СПИСОК ЛИТЕРАТУРЫ}

1. Каплинский, А. И. Об одном способе построения рандомизированных алгоритмов оптимизации / А. И. Каплинский, А. Е. Лимарев // Вопр. кибернетики: Случайный поиск в задачах оптимизации, 1978. - М. - С.13-17.

2. Каплинский, А. И. Построение рандомизированных алгоритмов оптимизации / А. И. Каплинский, А. Е. Лимарев, Г. Д. Чернышова // Проблемы случайного поиска. - Рига : Изд-во Зинатне, 1980. - Вып. 8. - С. 63-91.

3. Каплинский, А. И. Об одном способе построения рандомизированных алгоритмов / А. И. Каплинский, А. М. Песин. - М. : Из-во Автоматика и телемеханика, 1982. - № 12. C. $65-75$.

4. Каплинский, А. И. Вариационный под- 
ход к построению нелокальных алгоритмов оптимизации: препринт / А. И. Каплинский, А. И. Пропой. - М. : ВНИИСИ, 1986.

5. Каплинский, А. И. О градиентной основе нелокального поиска, использующего теорию потенциала / А. И. Каплинский, А. И. Пропой // Задачи и методы оптимального моделирования. - М. : ВНИИСИ, 1989.

6. Каплинскиц̆, А. И. Нелокальные алгоритмы поиска оптимальных решений / А. И. Каплинский, А. М. Песин // Оптимизация и моделирование в автоматизированных системах, 1991. - Воронеж : Изд-во Воронеж. политехн. ин-та. - С. 18-26.

7. Песин, А. М. Построение новых методов оптимизации на основе уравнений квантовой механики и математической физики / А. М. Песин. - Деп. ВИМИ, 28 февраля 1991 г., №ДО8340. - 30 c.

8. Каплинский, А. И. О методах нелокаль-

Каплинский А. И. - канд. техн. наук, доцент, доцент кафедры технической кибернетики и автоматического регулирования факультета ПММ Воронежского государственного университета.

Песин А. М. - канд. техн. наук, доцент, доцент кафедры технической кибернетики и автоматического регулирования факультета ПММ Воронежского государственного университета. ного поиска / А. И. Каплинский, А. М. Песин, А. И. Пропой // Модели и методы оптимизации. - М. : ВНИИСИ, 1991. - Вып. 13. - С. 3546.

9. Cybenko, G. Approximation by superpositions of a sigmoidal functions. - Univ. of Illinois, 1988.

10. Физика за рубежом // Сборн. статей. Серия «А». - М. : Мир, 1991.

11. Каплинскиц̆, А. И. Конструирование вычислительных алгоритмов нелокального поиска, использующих теорию потенциала / А. И. Каплинский, А. И. Пропой // препринт. - М. : ВНИИСИ, 1990. - 29 с.

12. Hunt, K. J. Neural networks for control systems - A Survey / K. J. Hunt, D. Sbarbaro, R. Zbikowski and P. J. Gawthrop // Automatica, 1992. - Vol. 28, No 6. - P. 1083-1112.

Kaplinskyi A. I. - Ph.D., Associate Professor, Department of Technical Cybernetics and Automatic Regulation, Voronezh State University.

Pesin A. M. - Ph.D., Associate Professor, Department of Technical Cybernetics and Automatic Regulation, Voronezh State University. 